\title{
Practical Three-Dimensional Profiling of Optical Fiber Preforms
}

\author{
PIERRE-LUC FRANCOIS, ISSEI SASAKI, AND M. J. ADAMS
}

\begin{abstract}
The spatial filtering technique has been used in the practical implementation of three-dimensional profile reconstruction for preforms of arbitrary cross section. An interpolation algorithm has been developed which enables accurate three-dimensional profiles to be obtained with a relatively modest number of azimuthal projections of the preform. With the aid of this algorithm it has been found, for both simulated and measured profiles, that for the majority of near-circular preforms only three projections need to be used; the storage requirements are now within the range of a minicomputer and the procedure is thus a practical tool for routine preform assessment.
\end{abstract}

\section{INTRODUCTION}

T HERE has recently been great interest in the development of nondestructive methods for accurately determining the refractive index profile in optical fiber preforms. Such methods usually involve the numerical inversion of the deflection function of the preform, i.e., the deflection angle of a ray in traversing the preform cross section as a function of the radial position of the ray and of the preform orientation [1]. The spatial filtering technique [2] has recently been shown to allow a quick and highly accurate measurement of the deflection function [3]. In this method a parallel beam of light illuminates the preform transversely and a rotating chopper converts the light deflection into measurable time-domain signals. Performing this measurement for several preform orientations permits three-dimensional reconstruction of the profiles for preforms of arbitrary cross section. The exceedingly large amount of data usually required for such reconstruction has been reduced by the development of a new interpolation algorithm which requires only a few azimuthal projections of the preform. The present paper describes this algorithm, analyzes the achievable accuracy, and gives results of three-dimensional reconstruction obtained for simulated and real preform profiles exhibiting a wide range of departures from circularity.

\section{THEORY}

Consider the situation of Fig. 1 where a preform of index profile $n(x, y)$, immersed in a matching fluid of refractive index $n_{o}$, is illuminated at an angle $\theta$ by a parallel beam of light.

Manuscript received August 31, 1981; revised November 12, 1981.

P.-L. Francois was on leave at the Department of Electronics, University of Southampton, Hampshire, England. He is with CNET, Lannion, France.

I. Sasaki and M. J. Adams are with the Department of Electronics, University of Southampton, Hampshire, England.

\section{A. Review of the Reconstruction Algorithms}

The well-known tomographic problem of determining a function $f(x, y)$, knowing the integrals $I_{f}(L)=\int_{L} f(x, y) d s$ of $f(x, y)$ along certain paths $L$, was first applied by Chu to optical fibers and preforms [1]. In this case $f(x, y)=n(x, y)-n_{o}$ and the integral $I_{f}(L)$ is the optical path length difference $\eta(\rho, \theta)$ between a ray traversing the path $A B$ through the preform and an imaginary ray traversing the same path but in the index matching fluid

$$
\eta(\rho, \theta)=\int_{L}\left(n(x, y)-n_{o}\right) d s
$$

where the line $L$ is the geometrical path $A B$ followed by the ray (Fig. 1).

The main hypothesis of the reconstruction is to assume that the optical fiber or preform may be regarded as a phase object [1]. This implies a perfect index match between the matching fluid and the fiber (or preform) cladding and also that we can approximate the actual ray trajectory $A B$ by the straight line $A B^{\prime}$ (Fig. 1); the latter hypothesis is justified for the usual range of relatively small numerical apertures encountered in practice. The straight line $A B^{\prime}$ is defined, as shown in Fig. 1, by the direction $\theta$ of the ray and the distance $\rho=\overline{O H}_{1}$.

In polar coordinates $(r, \psi)$, the index difference $\left(n(r, \psi)-n_{o}\right)$ of the reconstructed profile is then expressed in the following form [1]:

$$
\begin{aligned}
n(r, \psi)-n_{o}= & \frac{1}{2 \pi^{2}} \int_{-\pi / 2}^{\pi / 2} d \theta \int_{-\infty}^{+\infty} \frac{\partial \eta(\rho, \theta)}{\partial \rho} \\
& \frac{d \rho}{r \sin (\psi-\theta)-\rho} .
\end{aligned}
$$

For a detailed derivation of (2), we refer the reader to [1] where there is also an evaluation of the errors due to the ray curvature and to the index mismatch between the matching fluid and the cladding.

Equation (2) can be applied to both fibers and preforms; in the latter case, the path length difference $\eta(\rho, \theta)$ is difficult to measure and one uses the deflection $\phi(\rho, \theta)$ experienced by the ray $A B$ (Fig. 1). $\phi(\rho, \theta)$ can be obtained quickly and with high accuracy by the spatial-filtering technique reported elsewhere [2], [3]. $d \eta(\rho, \theta) / d \rho$ is then calculated numerically from $\phi(\rho, \theta)$ by the relation [4] 


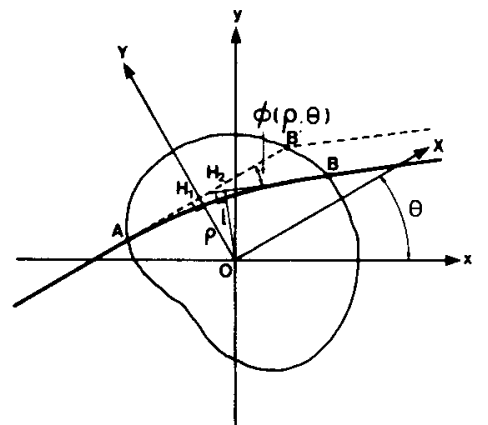

Fig. 1. Ray path $A B$ through a preform cross section, illustrating the straight line approximation $A B^{\prime}$. Solid line: actual ray path. Broken line: phase object approximation for the ray path.

$$
\frac{\partial \eta(\rho, \theta)}{\partial \rho}=n_{o} \phi(\rho, \theta)-n_{o} \frac{\partial}{\partial \rho}[\rho \phi(\rho, \theta)-l \tan [\phi(\rho, \theta)]]
$$

where $l=\overline{O H}_{2}$ in Fig. 1 .

As the deflection assumes very small values in practice, $\phi(\rho, \theta)$ differs only slightly from $\tan [\phi(\rho, \theta)]$ and the path length difference $\eta(\rho, \theta)$ is related to the deflection $\phi(\rho, \theta)$ in a very simple way $[5]$ :

$$
\frac{\partial \eta(\rho, \theta)}{\partial \rho} \simeq n_{o} \phi(\rho, \theta) \text {. }
$$

The approximation of (4), substituted into (2), gives the basic reconstruction expression for the normalized index difference:

$$
\frac{n(r, \psi)-n_{o}}{n_{o}}=\frac{1}{2 \pi^{2}} \int_{-\pi / 2}^{+\pi / 2} d \theta \int_{-\infty}^{+\infty} \phi(\rho, \theta) \frac{d \rho}{r \sin (\psi-\theta)-\rho}
$$

or, in the notation of [1]

$$
\frac{n(r, \psi)-n_{o}}{n_{o}}=\left.\frac{1}{2 \pi^{2}} \int_{-\pi / 2}^{+\pi / 2} d \theta g(z, \theta)\right|_{z=r \sin (\psi-\theta)}
$$

where

$$
g(z, \theta)=\int_{-\infty}^{+\infty} \phi(\rho, \theta) \frac{d \rho}{z-\rho}=\left[\phi(\rho, \theta) * \frac{1}{\rho}\right](z) .
$$

In (7) the asterisk denotes the convolution between $\phi$ and $1 / \rho$; in (6) $z=r \sin (\psi-\theta)$ is substituted in $g(z, \theta)$. A more rigorous formulation of (5)-(7) is given in Appendix A.

The reconstruction procedure has several steps as follows.

1) Using the spatial-filtering technique, the deflection function $\phi$ is measured for $N_{\theta}$ preform orientations, equally spaced in the range $\left(-90^{\circ}, 90^{\circ}\right)$. For each orientation, the whole preform width is scanned and the deflection is measured for $N_{\rho}$ values of $\rho$, with a scan-increment $\epsilon\left(N_{\theta}\right.$ is also called the number of projections).

2) The function $g(z, \theta)$ is tabulated; the array of the $g\left(z_{i}, \theta_{j}\right)$ is obtained from the array of the $\phi\left(\rho_{k}, \theta_{j}\right)$ by the convolution of (7). This convolution is best performed by the use of onedimensional FFT's [1].

3) For each point $(r, \psi)$ the normalized index difference is obtained by numerical integration of (6), in which the values of $g(z, \theta)$ are needed for $N_{I}$ values of $\theta$. The determination of $N_{I}$ will be discussed later.

For the special case of perfectly circular preforms, the explicit $\theta$-dependence of $\eta(\rho, \theta)$ and $\phi(\rho, \theta)$ disappears and we have two reconstruction equations, depending on whether we use either the path length difference $\eta(\rho)$ or the deflection function $\phi(\rho)$.

1) The Abel Transform Equation [5]:

$$
n(r)-n_{o}=-\frac{1}{\pi} \int_{r}^{\infty} \frac{d \eta(\rho)}{d \rho} \frac{d \rho}{\sqrt{\rho^{2}-r^{2}}} .
$$

2) The Marcuse Equation [7]:

$$
\frac{n(r)-n_{o}}{n_{o}}=-\frac{1}{\pi} \int_{r}^{\infty} \phi(\rho) \frac{d \rho}{\sqrt{\rho^{2}-r^{2}}} .
$$

We stress that (8) and (9) are intended only for use in the case of perfectly circular profiles.

\section{B. Practical Aspects of the Reconstruction}

For the sake of simplicity we now consider only the case of preforms but most of what is about to be said holds also for fibers. Two situations are to be distinguished.

1) "Every-Day" Near-CircularPreforms: Here the departure from perfect circularity, although always encountered in practice, is sufficiently small that a knowledge of the profile in only one section is adequate. The reconstruction of that section, however, must be a routine operation, by virtue of both simplicity and speed. For example the deflection measurement by the spatial-filtering technique takes about $5 \mathrm{~min}$ (with 1000 samples). As Marcuse's equation needs a further $5 \mathrm{~min}$, the complete reconstruction procedure takes therefore about 10 min (by Tektronix 4052).

2) Highly Asymmetric Preforms: Here a tomographic reconstruction must be used and the large amount of data corresponding to the projections $\phi(\rho, \theta)$ makes it essential to use a large computer. The measurement time increases considerably $(2 \mathrm{~h}$ $30 \mathrm{~min}$, for example, with 30 projections) and in addition there is the problem of data transfer to the computer, an operation which can take quite a long time. In this second situation, therefore, the reconstruction is no longer required to be a routine operation.

Let us consider the case 1) of quasi-circular preforms in more detail: as will be shown in Sections III and IV, the use of (9) sometimes gives rise to an unacceptable error even in this case. Therefore, a simplified 3-D reconstruction based on (6) would be useful in practice.

Assuming the most common departure from circularity to be an elliptical deformation, several attempts have been made to extend the usefulness of (8) and (9) by transforming the elliptically deformed profile into an "equivalent" circular one 
[6]- [8]. In Appendix B, we treat analytically the particular case of an elliptic preform with a parabolic index profile but the conclusions obtained are quite general and apply to any kind of profile function $f(u)$ [8]. We adopt the notation of Fig. 2(a) and consider the elliptical deformation of a circular profile

$$
\frac{n(u)-n_{o}}{n_{o}}=\Delta f(u)
$$

where $\Delta$ is the normalized index difference of the profile and

$$
u=\sqrt{\frac{x^{2}}{a^{2}}+\frac{y^{2}}{b^{2}}} \text { [with the notation of Fig. 2(a)]. }
$$

For a scan of the preform corresponding to the orientation $\theta$, the profile reconstruction from (9) is [8]

$$
\frac{n_{r}(r)-n_{o}}{n_{o}}=\Delta_{r} f\left(\frac{r}{R_{r}}\right)=\Delta \cdot \frac{a b}{R_{\theta}^{2}} f\left(\frac{r}{R_{\theta}}\right)
$$

where $R_{\theta}=\sqrt{a^{2} \sin ^{2} \theta+b^{2} \cos ^{2} \theta}$ is the apparent radius of the preform, seen in the direction $\theta$, and where subscript $r$ indicates the value obtained by reconstruction.

When the preform is scanned along a principal axis, $(\theta=0$ or $\pi / 2)$ the apparent radius corresponds to the actual one $\left(R_{\theta}=b\right.$ or $\left.a\right)$ whereas the normalized index difference $\Delta$ is multiplied (or divided) by the ratio $a / b$ [7] [if $\theta=0, \Delta_{r}=$ $\Delta(a / b)$ and if $\left.\theta=\pi / 2, \Delta_{r}=\Delta(b / a)\right]$. The orientation of the principal axes can easily be found by observing an image of the core as the preform is being rotated; from scans in these two directions the ratio $a / b$ can be evaluated and both the piofile function $f(u)$ and the actual value of $\Delta$ can be determined [7], [8].

Using this method, elliptically deformed profiles can thus be measured just as easily and just as quickly as those of circular preforms without the need for using three-dimensional reconstructions [7]. Unfortunately, the method of "equivalent profile" cannot be extended to other kinds of deformation and is therefore of limited use.

Owing to the explicit $\theta$-dependence of $g(z, \theta)$, each value of $\theta$ refers to a different projection in the numerical integration of (6). In theory it would thus be necessary to measure as many projections as there are $\theta$-values considered in the integration. However, in most practical cases the azimuthal preform variations are always very smooth and we have applied (5) with only a few measured deflections $\phi(\rho, \theta)$, using interpolated values of the convolution $g(z, \theta)$. This interpolation technique is justified also, as is shown in Appendix A, by the fact that (5) reduces to (9) in the ideal case of perfectly symmetrical preforms. Interpolation provides an intermediate solution between (9), using only one projection, and (5), applied with a large number of projections. In most cases a good profile reconstruction can be obtained with only three projections. The memory requirements corresponding to three projections are within the storage capacity of a laboratory minicomputer.

For stronger departure from circularity more projections are needed but, as the azimuthal variations remain smooth, the total number of projections remain small (5-13), thus allow-

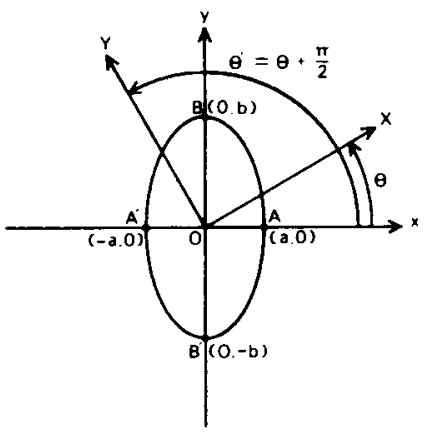

(a)

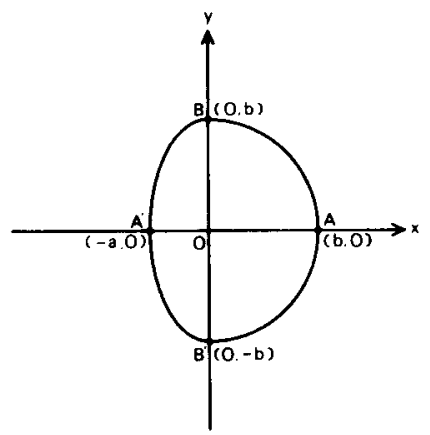

(b)

Fig. 2. Core deformations considered in the simulations. (a) Elliptical core deformation. (b) Half-elliptical core deformation.

ing moderate times for measurement and data transfer. It was never necessary to measure more than 21 projections, even in the extreme case of the very elliptical preform shown in Section IV. However, it is worth noting that in the case of strong azimuthal dependence of CVD layer structure it may, of course, be necessary to use more projections to ensure the fine detail of the 3-D reconstruction is not omitted. Such cases are sometimes observed for the innermost layers in multimode fiber preforms prepared by the OVPO process.

The first decision one has to make in practice is the number $N_{\theta}$ of projections to use. We distinguish here three different cases:
1) one projection is measured
2) three projections are measured $\}$ where processing is within the capability of a minicomputer
3) more than three projections are measured.

Sections III and IV will determine the decision thresholds between these cases for given allowed error levels.

The second practical parameter is the scan-increment $\epsilon$. The number of points at which the deflection must be known is determined by the sampling theorem, according to which most of the spectral power must be contained in the frequency interval $[-1 / 2 \epsilon, 1 / 2 \epsilon]$. However, it is usually sufficient to empirically select an increment $\epsilon$ small enough to describe sufficiently well the smallest preform details. Practical values of $\epsilon$ range from 4-10 $\mu \mathrm{m}$.

The two previous parameters determine the time for measurement and data transfer. In the reconstruction they modify only the convolution calculation time, always very short, and have therefore no critical influence in the total computing time.

The parameter determining the computing time for one 
point of index profile is the number $N_{I}$ of $\theta$-values taken in the integration. If $N_{I}$ equally spaced $\theta$-values are used, the corresponding $z$-variation is of the order of $\Delta z \simeq|r| \Delta \theta=$ $|r| \pi / N_{I}$. It appears therefore that, for a given $N_{I}$, details of the index profile smaller than $\Delta z$ are missed; in other words, the reconstruction resolution is of order $|r| \pi / N_{I}$, at a distance $|r|$ from the center. The resolution is thus always better near the center, i.e., for $|r|$ small, and it is possible to improve the resolution by increasing $N_{I}$. Near the center, a given resolution can be obtained with a reduced $N_{I}$. The resolution is, however, limited by the accuracy of the deflection measurement and in any case cannot be better than the scan increment $\epsilon$. Augmenting $N_{I}$ above $|r| \pi / \epsilon$ increases the computing time without increasing the reconstruction resolution. Practical values of $N_{I}$ range from $50-500$.

Any kind of interpolation routine can be used to determine the values of $g(z, \theta)$ from the array of the $g\left(z_{i}, \theta_{j}\right)$; in practice, the two-dimensional interpolation involved is best performed by using two one-dimensional interpolations. As the points $\left(z_{i}, \theta_{j}\right)$ for which the convolution $g(z, \theta)$ is known are situated on a regular grid, a Lagrange-type interpolation can be used [9]. The difference between two $z_{i}$ is equal to the scan increment $\epsilon$, which is always relatively small, and thus in the $z$ direction we have used a Lagrange interpolation involving only three points. The difference between two $\theta_{j}$ (equal to $\pi / N_{\theta}$ ) is much larger and we have used a six-point Lagrange interpolation. Even if the number $N_{\theta}$ of projections measured is three, it is possible to use an interpolation formula involving six values of $\theta$ because of the periodicity $g(z, \theta)=g(z, \theta+\pi)$. This period. icity can easily be demonstrated from the definition of $g(z, \theta)$ in (7), under the assumption that the preform may be regarded as a phase object.

\section{Results for Simulated Profiles}

Using numerical ray tracing, we simulate the set of deflection functions $\phi(\rho, \theta)$ corresponding to original profiles of the form

$$
\begin{array}{ll}
n^{2}(x, y)=n_{1}^{2}\left\{1-2 \Delta\left[\left(\frac{x}{a_{1}}\right)^{2 p_{1}}+\left(\frac{y}{b}\right)^{2}\right]\right\} & \text { for } x \leqslant 0 \\
n^{2}(x, y)=n_{1}^{2}\left\{1-2 \Delta\left[\left(\frac{x}{a_{2}}\right)^{2 p_{2}}+\left(\frac{y}{b}\right)^{2}\right]\right\} & \text { for } x>0
\end{array}
$$

where we always choose

$$
\Delta=0.01
$$$$
n_{1}=1.5=n_{o}(1+\Delta)
$$

$n_{o}=$ refractive index of the matching fluid

$p_{1}$ and $p_{2}$ are power-law exponents describing the profile deformation.

The deflection is calculated with an increment $\epsilon$ for each of the $N_{\theta}$ equally spaced $\theta$-values. The numerical integration of (6) is performed with $N_{I}$ points.

Depending on the case under consideration, the reconstruction quality will be characterized by parameters chosen from the following three.
1) Index-difference error: $\left|\frac{\Delta_{r}-\Delta}{\Delta}\right|$
(in percent)

2) Maximum index error with respect to the index-difference $n_{o} \Delta$ :

$$
\max _{(x, y)}\left|\frac{n_{r}(x, y)-n(x, y)}{n_{o} \Delta}\right| \quad \text { (in percent) }
$$

3) Mean index error with respect to the index-difference $n_{o} \Delta$ :

$$
\underset{(x, y)}{\operatorname{mean}}\left|\frac{n_{r}(x, y)-n(x, y)}{n_{o} \Delta}\right| \quad \text { (in percent) }
$$

where the subscript $r$ indicates the value obtained by reconstruction.

We consider the effect on the reconstruction quality of the scan-increment $\epsilon$, of the number $N_{I}$ of points in the integration of (6) and, lastly, of the number $N_{\theta}$ of projections. For the latter parameter, the discussion will concern the reconstruction degradation when using Chu's equation (6) with only a few projections and the limit of applicability of Marcuse's equation for a given error level.

\section{A. Scan-Increment $\epsilon$}

A circular preform with parabolic index profile was assumed $\left(a_{1}=a_{2}=b=1 \mathrm{~mm} ; p_{1}=p_{2}=1\right)$ and the deflection $\phi(\rho)$ was simulated with increments $\epsilon$ ranging from 100 to $10 \mu \mathrm{m}$. The integration of (6) [which reduces in the circular case to (9)] was performed with 441 points in order to ensure that the accuracy was limited by $\epsilon$, and not by $N_{I}$ being chosen too small $\left(N_{I}=441>\max |r| \pi / \epsilon \simeq 314\right)$. The resulting values of the maximum and of the average index error are shown in Table I.

In [1] measurement data are regarded as a band-limited signal with bandwidth $\Omega=5 /$ core radius. To satisfy the sampling theorem we must have $\Omega=5 /$ core radius $<1 / 2 \epsilon$ which gives a minimum number of 20 measurements across the preform cross section [1]. Twenty measurements correspond here to $\epsilon=100 \mu \mathrm{m}$ and Table I shows the increased accuracy obtained when taking more than that minimum number.

\section{B. Number $N_{I}$ of Integration Points}

We consider the circular preform of Section III-A but the scanincrement is fixed now at $\epsilon=10 \mu \mathrm{m}$, corresponding to 200 samples across the preform cross section. Table II shows the maximum and the average index error found for arbitrary values of $N_{I}$ ranging from 21 to 441 . The reconstruction accuracy is no longer improved for $N_{I}>\pi \cdot 1 \mathrm{~mm} / 10 \mu \mathrm{m}=314$, as in dicated in Section II, and has almost achieved its limit value for $N_{I} \simeq$ 100.

\section{Effect of Interpolations in Chu's Equation}

The number $N_{\theta}$ of projections to be measured is determined by the rate of the azimuthal variations; as illustrations we consider the four following cases of departure from a circular preform.

\section{1) Elliptical Core Deformation:}

$$
\begin{aligned}
& p_{1}=p_{2}=1 \\
& a_{1}=a_{2}=a \leqslant b
\end{aligned}
$$$$
\text { deformation rate }=e=\frac{b-a}{b} \text {. }
$$ 
TABLE I

Effect of the Scan-Increment $\epsilon$ on the Reconstruction Accuracy. The Errors are Expressed as as a Percentage of the Index DIFFERENCE $n_{0} \Delta$.

\begin{tabular}{|c|c|c|}
\hline$\varepsilon(\mu \mathrm{m})$ & $\begin{array}{c}\text { Maximum } \\
\text { index-error } \\
(8)\end{array}$ & $\begin{array}{c}\text { Average } \\
\text { index-error } \\
(8)\end{array}$ \\
\hline 100 & 4.8 & 2.2 \\
80 & 2.8 & 1.5 \\
60 & 1.4 & 0.9 \\
40 & 0.7 & 0.5 \\
10 & 0.43 & 0.24 \\
\hline
\end{tabular}

TABLE II

Effect of the Number $N_{I}$ of Integration Points on the Reconstruction Accuracy. The Errors are Expressed as a Percentage of THE INDEX DIFFERENCE $n_{0} \Delta$.

\begin{tabular}{|c|c|c|}
\hline $\mathrm{N}_{\mathrm{I}}$ & $\begin{array}{c}\text { Maximm } \\
\text { index-error } \\
(8)\end{array}$ & $\begin{array}{c}\text { Average } \\
\text { index-error } \\
(8)\end{array}$ \\
\hline 21 & 1.5 & 1.1 \\
63 & 0.46 & 0.25 \\
105 & 0.45 & 0.245 \\
147 & 0.44 & 0.241 \\
231 & 0.436 & 0.241 \\
273 & 0.43 & 0.241 \\
357 & 0.43 & 0.241 \\
399 & 0.43 & 0.241 \\
441 & 0.43 & 0.241 \\
\hline
\end{tabular}

2) Half-Elliptical Core Deformation:

$$
\begin{aligned}
& p_{1}=p_{2}=1 \\
& a_{2}=b \\
& a_{1}=a \leqslant b
\end{aligned}
$$

deformation rate $=e=\frac{b-a}{b}$.

\section{3) Elliptical Index Deformation:}

$$
\begin{aligned}
& p_{1}=p_{2}=p \leqslant 1 \\
& a_{1}=a_{2}=b
\end{aligned}
$$

deformation rate $=e=1-p$.

4) Half-Elliptical Index Deformation:

$$
\begin{aligned}
p_{1} & =1 \\
p_{2} & =p \leqslant 1 \\
a_{1} & =a_{2}=b \\
\text { deformation rate } & =e=1-p .
\end{aligned}
$$

Cases 1) and 2) are illustrated in Fig. 2(a) and (b), respectively. As stressed in Section II, we have three reconstruction possibilities: 1) for very small deformations, use of Marcuse's equa- tion (9); 2) for small deformations, use of (6) with only three projections; 3 ) use of (6) with more than three projections. Reconstructions 1) and 2) are within the capability of a minicomputer.

In this section, we illustrate cases 1) and 2) and consider the degradation suffered by the reconstruction when the $N_{I}$ points needed in the integration of (6) are obtained from a reduced number $N_{\theta}$ of projections. To that end, the deflection functions $\phi(\rho, \theta)$ corresponding to the four above-mentioned deformations were simulated with a scan-increment $\epsilon=10 \mu \mathrm{m}$ and with $N_{\theta}=21$. By selecting, from that set of 21 projections, every third and every seventh projection, we obtained the sets corresponding to $N_{\theta}=7$ and $N_{\theta}=3 . N_{I}$ was taken equal to $21 \times 21=441$ and the number of interpolations between each projection was

$$
\begin{array}{r}
21 \text { for } N_{\theta}=21 \\
63 \text { for } N_{\theta}=7 \\
147 \text { for } N_{\theta}=3 .
\end{array}
$$

The interpolation routine used was described at the end of Section II.

For deformations 1-4, Table III shows the maximum normalized error we found as a function of the deformation rate and for $N_{\theta}=21,7,3$ : the results for $N_{\theta}=21$ and 7 are rather close and an enhanced degradation occurs only in the case $N_{\theta}=3$. The nonsymmetrical deformations 2 and 4 amplify the degradation more than the symmetrical ones 1 and 3 .

If one tolerates a maximum index difference error of 5 percent, for example, then Table III shows that the minicomputer reconstruction with $N_{\theta}=3$ can be used for core ellipticities up to 16 percent and for index deformations in the order of 20 percent.

\section{Applicability of Marcuse's Equation}

We turn now to the case of very small deformations and investigate the limit of applicability of (9). The deflection function $\phi(\rho)$ associated with a perfectly circular preform is an antisymmetrical function of $\rho(\phi(\rho)=-\phi(-\rho))$ and (9) uses only the values of $\phi$ for $\rho \geqslant 0$. In the measurement process, however, the whole preform is scanned and the deflection is obtained for values of $\rho$ both positive and negative. In practice, (9) can thus be applied successively to the left and right half of the deflection $(-\phi(\rho))$ with $\rho \leqslant 0$ and $\phi(\rho)$ with $\rho \geqslant 0$, respectively) and two index profiles are obtained: they only coincide in the case where $\phi$ is a strictly antisymmetrical function of $\rho$. As will be shown, the discrepancy between these two profiles depends on the preform orientation for which the deflection was measured; taking the average of these two profiles is justified to some extent in Appendix A and can sometimes yield better results. There are cases, however, where the two profiles are exactly identical but do not correspond, for all that, to the actual one.

To express the reconstruction quality, we had to choose a parameter having the same definition for the circular profile, given by (9), and the actual deformed one; hence we consider here only errors in index difference. 
TABLE III

Errors Incurred by Interpolation in Chu's Equation for Reconstruction (5). Four Cases of Basic Deformation are Considered. The Errors are Again Normalized to the Index DIFFERENCE $n_{0} \Delta$

\begin{tabular}{|c|c|c|c|c|c|c|c|c|c|c|c|c|}
\hline & \multicolumn{3}{|c|}{$\begin{array}{l}\text { elliptical } \\
\text { orre } \\
\text { deformation }\end{array}$} & \multicolumn{3}{|c|}{$\begin{array}{c}\text { half-elliptical } \\
\text { core } \\
\text { deformation }\end{array}$} & \multicolumn{3}{|c|}{$\begin{array}{c}\text { elliptical } \\
\text { index } \\
\text { deformation }\end{array}$} & \multicolumn{3}{|c|}{$\begin{array}{l}\text { half-elliptical } \\
\text { index } \\
\text { deformation } \\
\end{array}$} \\
\hline & & $\mathrm{N}_{\theta}$ & & & $\mathbf{N}_{\theta}$ & & & $\mathrm{N}_{\Theta}$ & & & $\mathrm{N}_{\Theta}$ & \\
\hline & 21 & 7 & 3 & 21 & 7 & 3 & 21 & 7 & 3 & 21 & 7 & 3 \\
\hline \multirow{5}{*}{ 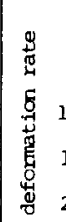 } & 0.5 & 0.75 & 1.2 & 0.6 & 1.1 & 2 & 0.5 & 0.52 & 1.16 & 0.5 & 0.55 & 1.25 \\
\hline & 0.6 & 0.9 & 2 & 0.7 & 1.8 & 4.2 & 1. 52 & 0.68 & 2 & 0.54 & 0.7 & 2.2 \\
\hline & 0.7 & 1.1 & 3.3 & 0.8 & 2.7 & 7 & 0.56 & 0.88 & 2.76 & 0.6 & 1 & 3 \\
\hline & 0.8 & 1.5 & 4.8 & 1.0 & 3.8 & 10 & 0.6 & 1.32 & 3.72 & 0.7 & 1.5 & 4.2 \\
\hline & 0.9 & 2.4 & 7 & 1.3 & 5 & 13.8 & 0.65 & 1.84 & 5 & 0.84 & 2.3 & 6 \\
\hline
\end{tabular}

TABLE IV

ERrors in Marcuse's Reconstruction for Four CASES OF Deformation. Column I: Maximum Error. Column II: Maximum Error When the Average Index Difference IS CONSIDERED.

\begin{tabular}{|c|c|c|c|c|c|c|c|c|}
\hline & \multicolumn{2}{|c|}{$\begin{array}{l}\text { elliptical } \\
\text { core } \\
\text { deformation }\end{array}$} & \multicolumn{2}{|c|}{$\begin{array}{c}\text { half-elliptical } \\
\text { core } \\
\text { deformation }\end{array}$} & \multicolumn{2}{|c|}{$\begin{array}{l}\text { elliptical } \\
\text { index } \\
\text { deformation }\end{array}$} & \multicolumn{2}{|c|}{$\begin{array}{c}\text { half-elliptical } \\
\text { index } \\
\text { deformation }\end{array}$} \\
\hline 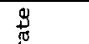 & I & II & I & II & I & II & I & II \\
\hline & 4.16 & 4.16 & 4.16 & 2.08 & 4.4 & 4.4 & 4.4 & 2.2 \\
\hline हु & 8.64 & 8.64 & 8.64 & 4.32 & 9.4 & 9.4 & 9.4 & 4.7 \\
\hline$\underset{J}{J} 128$ & 13.44 & 13.44 & 13.44 & 6.72 & 15 & 15 & 15 & 7.5 \\
\hline
\end{tabular}

Table IV shows the results of a simulation for the deformations 1-4. The deflection was calculated for $\theta$-values in the interval $\left(-90^{\circ}, 90^{\circ}\right)$ and for each $\theta$ Marcuse's equation was applied to the left and the right half of the deflections $\phi(\rho, \theta)$, thus giving two index differences $\Delta_{L}$ and $\Delta_{R}$. Table IV reports the maximum error in index difference together with the error made when the index difference $\left(\Delta_{L}+\Delta_{R}\right) / 2$ of the average profile is considered. Considering the average profile improves the accuracy by a factor of two with nonsymmetrical deformations 2 and 4 but does not provide any improvement in the symmetric cases 1 and 3 . To explain this effect, we describe the elliptical and the half-elliptical core deformation in more detail.

In the case of the elliptical core deformation of a small numerical aperture preform, the deflection function $\phi(\rho, \theta)$ is nearly antisymmetrical for any preform orientation:

$$
\phi(\rho, \theta) \simeq-\phi(-\rho, \theta) \quad \forall \theta .
$$

For each $\theta$, two profiles are obtained by the application of (9) to the two halves $\rho \leqslant 0$ and $\rho \geqslant 0$ of the deflection; they are identical in this particular case and taking the average does not reduce the error, as is shown in Table IV. From Appendix $\mathrm{B}$, the error in index difference for this case can be expressed as

$$
\begin{aligned}
\operatorname{error}(\Delta) & =\frac{\Delta_{r}-\Delta}{\Delta}=e \cos 2 \theta^{\prime}+e^{2} \cos \theta^{\prime} \cos 3 \theta^{\prime}+0\left(e^{3}\right) \\
& =e \cos 2 \theta^{\prime} \quad\left(\text { to order } e^{2}\right)
\end{aligned}
$$

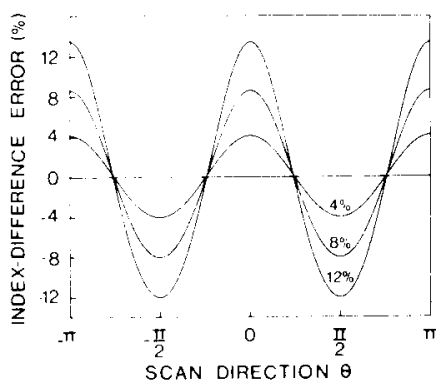

(a)

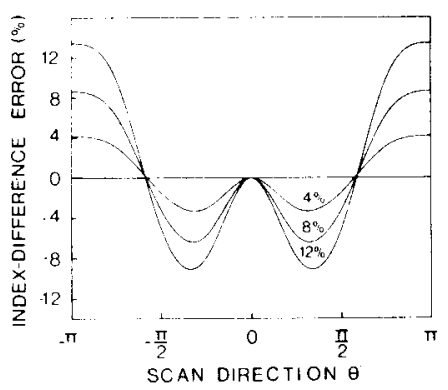

(b)

Fig. 3. Error caused by the use of Marcuse's equation in case of a core deformation, as a function of the scan-direction $\theta^{\prime}$. (a) Elliptical core deformation. (b) Half-elliptical core deformation. Labeling parameter indicates the deformation rate.

where $e=$ deformation rate $=(b-a) / b$, and $\theta^{\prime}$ is the scan-direction, as is shown in Fig. 2(a).

The error distribution as a function of the scan direction $\theta^{\prime}$ is shown in Fig. 3(a). We stress that the maximum error occurs for $\cos 2 \theta^{\prime}= \pm 1$ corresponding to scans along the principal axis of the ellipse; a scan along the minor axis, for which $\theta=0$ or $\pi$, yields too high an index difference (error $(\Delta) \simeq+e$ percent) whereas a scan along the major axis $\left(\theta^{\prime}= \pm \pi / 2\right)$ yields too small a result (error $(\Delta) \simeq-e$ percent). A scan direction of $\theta^{\prime}= \pm \pi / 4$ gives the right answer (to order $e^{2}$ ).

Similarly, the error distribution corresponding to a halfelliptical core deformation is shown in Fig. 3(b). Here the maximum error corresponds to a scan along $A^{\prime} O A$ [Fig. 2(b)] . The profile reconstructed from the scan along $O A\left(\theta^{\prime}=0\right)$ has the actual index difference whereas the use of the scan along $O A^{\prime}\left(\theta^{\prime}= \pm \pi\right)$ yields too high a result (with an error $+e$ percent); in the case of a scan along $A^{\prime} O A$, taking the average therefore improves the accuracy by a factor of exactly two. Other results are found for other scan directions (no improvements, for example, with a scan along $B^{\prime} O B$ ) but the maximum error when averaging corresponds always to the scan along $A^{\prime} O A$, which explains the improvement by the factor of exactly two observed in Table IV.

If one tolerates an arbitrary error level of 5 percent, Table IV shows that Marcuse's equation can be used for deformations of any kind up to about 5 percent. For the two asymmetric deformations 2 and 4 , the use of averaging extends that limit to 8 percent.

It is possible to extend this method of averaging to more than two profiles by scanning the preform in several directions. In the case of an elliptical core deformation, for example, (11) shows that taking the average of the results corresponding to the two principal axes of the ellipse $\left(\theta^{\prime}=0\right.$ and $\left.\theta^{\prime}=\pi / 2\right)$ gives the right result (to order $e^{2}$ ). We must stress, however, 
that if one wants to measure more than one projection, the best utilization of the data is to employ them in a reconstruction with Chu's equation (Section III-C).

\section{Results for Measured Profiles}

Four preforms are used to illustrate the several points stressed in the two previous sections; the first three, $A, B$, and $C$, are quasi-circular and the fourth, $D$, is highly elliptical. The composition of these preforms are as follows:

$A$ : fluorophosphosilicate cladding, germanosilicate core

$B$ : phosphosilicate cladding, germanosilicate core

$C$ : borosilicate cladding, germanosilicate core

$D$ : fluorophosphosilicate cladding, germanosilicate core.

For more detail, we show an index profile section of each preform [Fig. 4(a)-(d)] together with a three-dimensional display; the core and the complete cladding of preform $A$ are shown in Fig. 5(a) but, for preforms $B$ and $C$, we have only shown a view of the core and of the four inner cladding-layers [Fig. 5(b) and (c)]. The core of the highly elliptical preform is displayed in Fig. 5(d) and the section of Fig. 4(d) corresponds to the major axis.

Following the progression of Section III, we consider successively the choice of the scan-increment $\epsilon$, the number $N_{I}$ of integration points in (6), and the projection number $N_{\theta}$. The discussion concerning $N_{\theta}$ is devoted mainly to the reconstruction degradation when using Chu's equation with only a few projections and to the applicability of Marcuse's equation.

\section{A. Scan-Increment}

In practice, as mentioned in Section II, $\epsilon$ is chosen empirically, the rule being to describe the preform details with a sufficient number of samples. Quite arbitrarily we have always taken at least ten samples per detail in our measurements.

The deflection function of preform $B$ is shown in Fig. 6 for a value of $\theta$ and, in this case, the layer structure has a periodicity of about $50 \mu \mathrm{m}$, near the cladding-substrate boundary. To obey our rule, the scan-increment $\epsilon$ was fixed at $\epsilon=4.8 \mu \mathrm{m}$. For preform $A$, which has no layer-structure, $\epsilon=10 \mu \mathrm{m}$ was taken. Preform $C$ was measured with $\epsilon=4 \mu \mathrm{m}$ and the highly elliptical one, $D$, with $\epsilon=6 \mu \mathrm{m}$. We have measured a large number of other preforms and have always selected the scan-increment $\epsilon$ in the range $4-10 \mu \mathrm{m}$.

\section{B. Number $N_{I}$ of Integration Points}

As pointed out in Section II, the resolution, at a distance $|r|$ from the center, is in the order of magnitude of $\Delta z=|r| \pi / N_{I}$; as we concluded there, the reconstruction is always better near the axis origin, corresponding to small values of $|r|$. A comparison of Fig. 7(a), (b) and Fig. 5(a) illustrates the phenomenon; preform $A$ is displayed in each plot and the only different param. eter is $N_{I}$. Preform $A$ was measured with 13 projections and in Fig. 7 (a) $N_{I}=13$ only: we observe waves along 13 diameters corresponding to the 13 equally spaced preform orientations of the deflection measurement. In Fig. 7(b) and Fig. 5(a) we have increased $N_{I}$ by interpolation: in Fig. 7(b), $N_{I}=13 \times 3=$ 39 and the radial waves are only present in the substrate region.

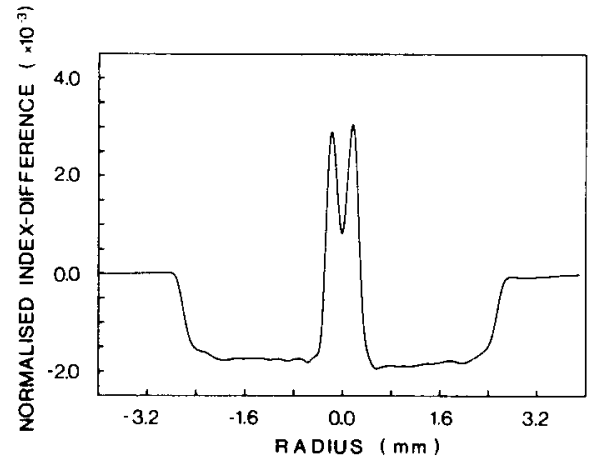

(a)

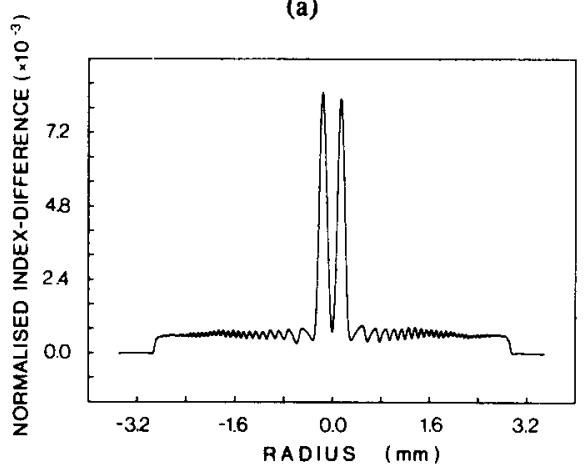

(b)

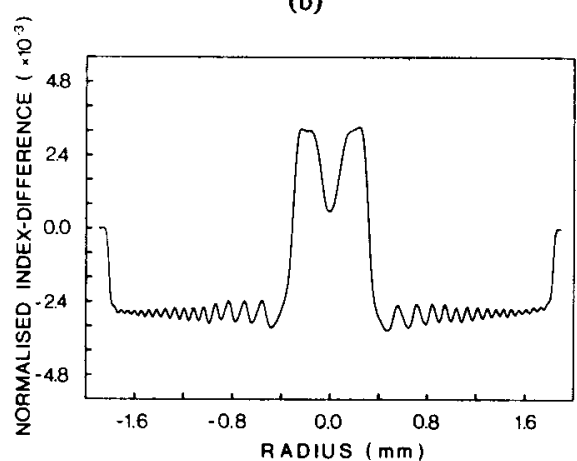

(c)

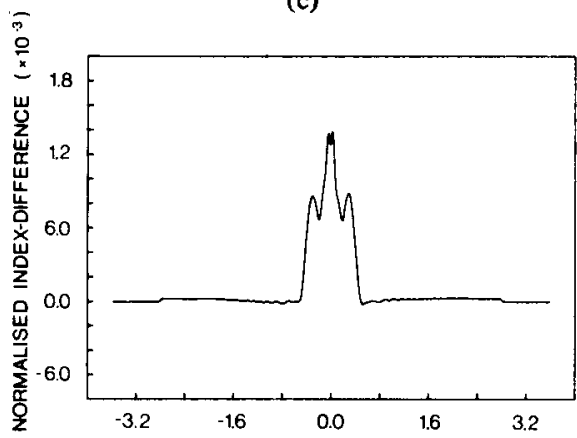

(d)

Fig. 4. Index profile section of preforms $A$ (a), $B(\mathrm{~b}), C$ (c), and of the major axis of preform $D$ (d). The profiles of preforms $B$ and $C$ were reconstructed with only three projections.

In Fig. 5(a) where $N_{I}=13 \times 9=117$, the waves have entirely disappeared. These three figures illustrate clearly the kind of spurious wave distribution which arises when the integration of (5) is not performed with a sufficient number $N_{I}$ of points; they confirm also that the degradation is always smaller near the center. 


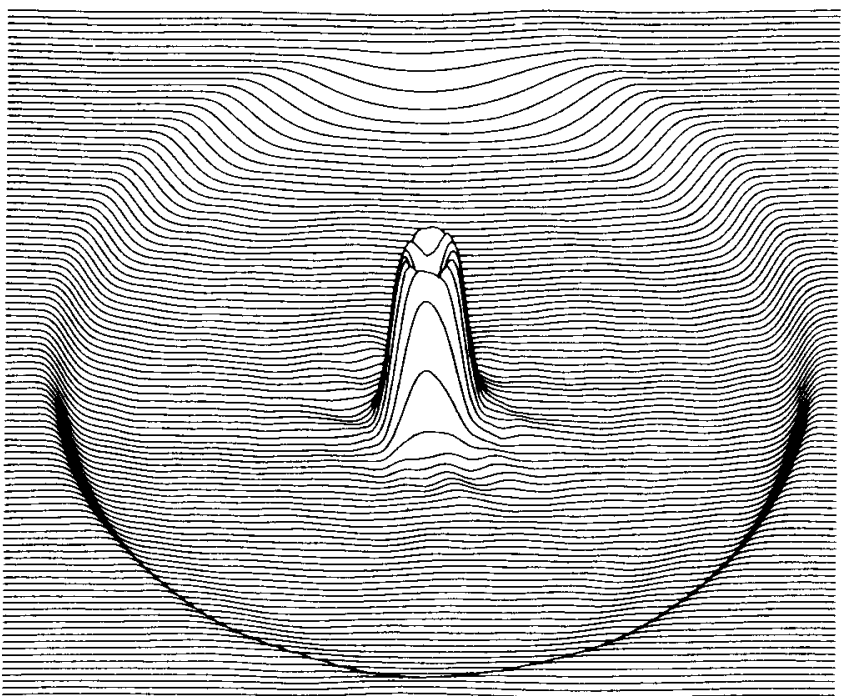

(a)

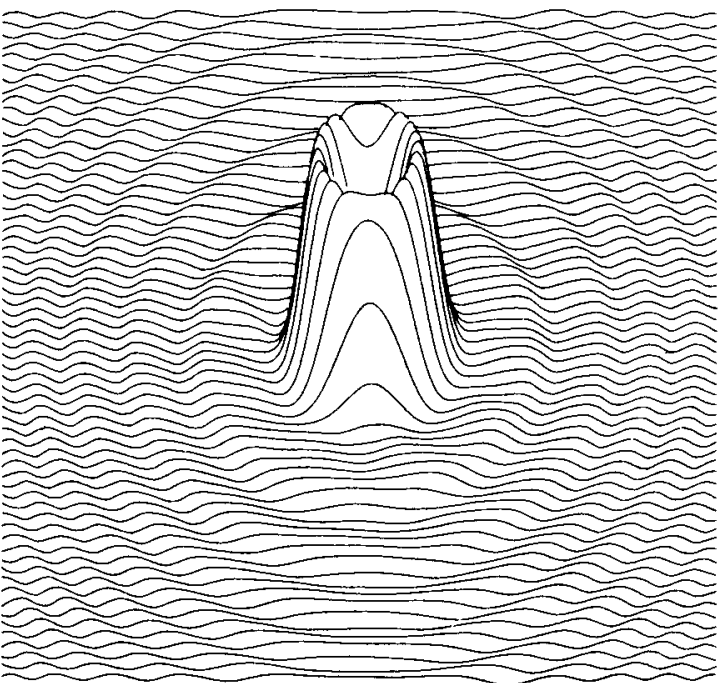

(b)

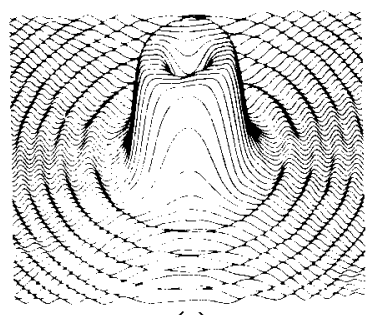

(c)

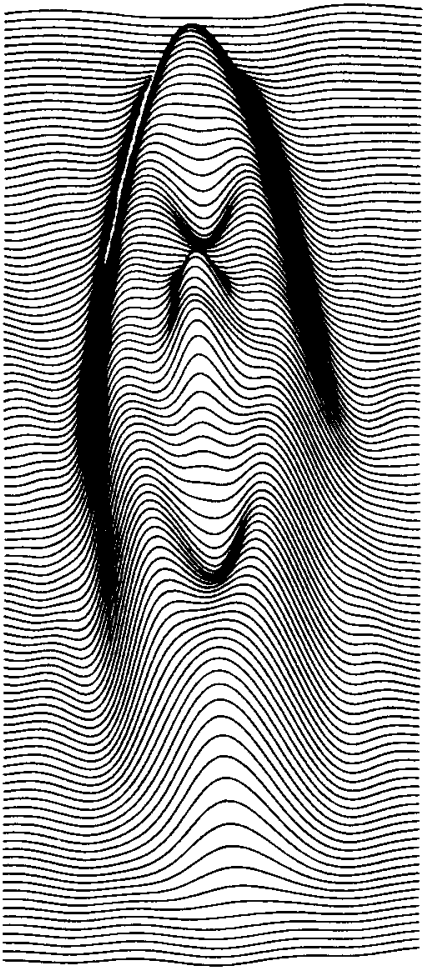

(d)

Fig. 5. Three-dimensional display of preforms $A$ (a), of the core and of the four inner cladding layers of preforms $B$ (b), and $C(\mathrm{c})$, and of the core of preform $D(\mathrm{~d})$.

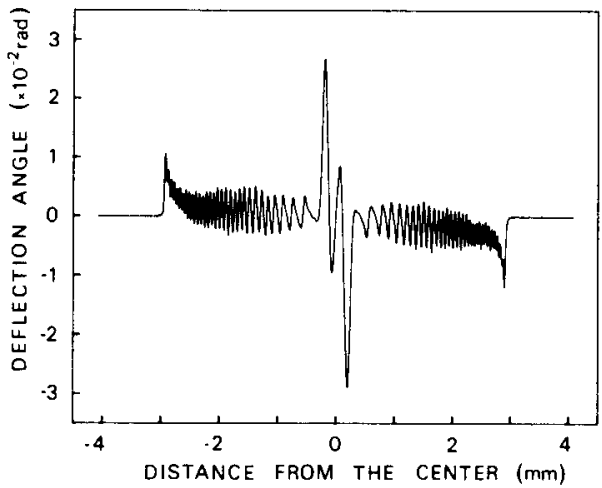

Fig. 6. Defleciion function of preform $B$.

Reciprocally, for the reconstruction of index points situated near the center, a smaller number $N_{I}$ can provide the desired accuracy. Fig. 5(b), (c), and (d), which only display the core region of preforms $B, C$, and $D$, were obtained with $N_{I}=21 \mathrm{X}$ $11=231$ whereas the sections of Figs. 4(b), (c), and (d) were calculated with $N_{I}=21 \times 19=399$ (preforms $B, C$, and $D$ were all measured with $N_{\theta}=21$ projections).

\section{Effects of Interpolation in Chu's Equation}

In this section and the next one we come to the problem of choosing the reconstruction algorithm. In Section II we have distinguished three possibilities and the simulations of Section III have determined decision thresholds between them (for an arbitrary maximum error level of 5 percent). What happens in practice will be illustrated with preforms $B, C$, and $D$ (all measured with 21 projections).

In the case of measured preforms we no longer know the exact profile, as was the case for the simulations. We assume the exact profile to be provided by the reconstruction with the 21 projections and all the index errors are related to that profile, 


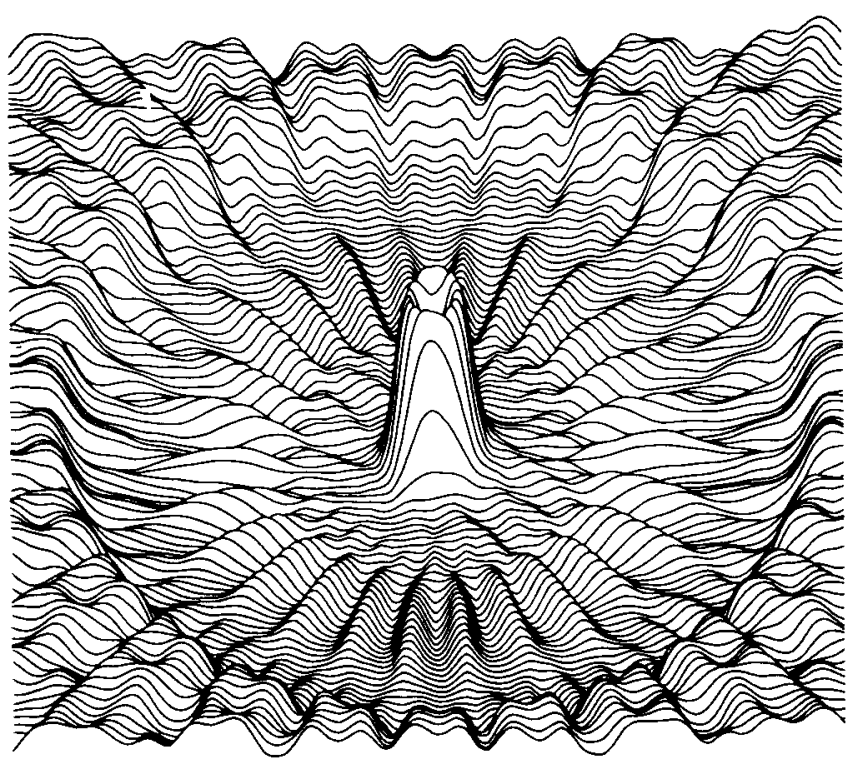

(a)

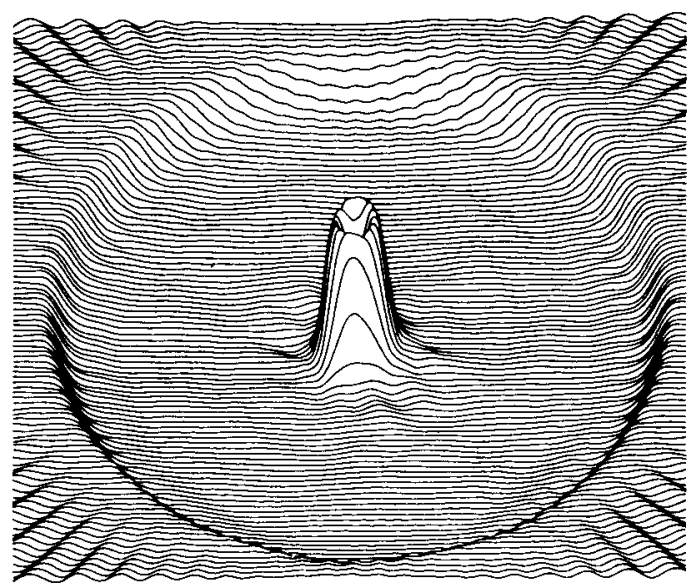

(b)

Fig. 7. Illustration of the effect of taking too small a number $N_{I}$ of $\theta$ values in the integration of (6) for preform $A$. (a) No interpolation, $N_{I}=13$. (b) $N_{I}=13 \times 3=39$.

taken as reference. To give meaning to the comparison between the profiles obtained with 3,7 , and 21 projections, and to eliminate the effect of the experimental conditions, the preforms were measured only once and the sets of 3 and 7 projections were obtained from the 21 in the way described in Section III-C.

Table $\mathrm{V}$ compares the reconstruction with 3 and 7 projections for preforms $B$ and $C$. The index difference error, the maximum index error and the average index error, expressed in percent, are normalized by the preform index difference (for preform $B, \Delta=0.00853$ and for preform $C, \Delta=0.00665$ ).

For an arbitrary 5 percent level of maximum error, the 3 projections minicomputer reconstruction can be used with preform $B$ but not with preform $C$. We have tried to relate the results of Table $\mathrm{V}$ to the simulations of Section III and have attributed the reconstruction degradation to an indexdeformation. The cores of preforms $B$ and $C$ were found to be perfectly circular but, as is seen in the three-dimensional displays of Fig. 5(b) and (c), some kind of index asymmetry occurs. The effect is very pronounced for preform $C$ and explains the enhanced degradation we have observed. In the two
TABLE $V$

Comparison of Results from Chu's Equation When Usinr 7 and 3 Projections, Assuming the Result with 21 Projections Corresponds to the Actual Profile

\begin{tabular}{|c|c|c|c|c|c|c|}
\cline { 2 - 7 } \multicolumn{1}{c|}{} & \multicolumn{2}{c|}{$\begin{array}{c}\text { Index-difference } \\
\text { error }(8)\end{array}$} & \multicolumn{2}{|c|}{$\begin{array}{c}\text { Maximam } \\
\text { Index-difference } \\
\text { error ( }(8)\end{array}$} & \multicolumn{2}{|c|}{$\begin{array}{c}\text { Average } \\
\text { Index-difference } \\
\text { error (8) }\end{array}$} \\
\hline $\mathrm{N}_{\Theta}$ & 7 & 3 & 7 & 3 & 7 & 3 \\
\hline $\mathrm{B}$ & 0.14 & 0.24 & 2.5 & 3.7 & 0.42 & 0.44 \\
\hline $\mathrm{C}$ & 1 & 1.8 & 3.6 & 5.8 & 0.65 & 0.84 \\
\hline
\end{tabular}

cases of preforms $B$ and $C$, the degradation does not distort the profile-shape. As a matter of fact, the index sections of Figs. 4(b) and (c) were obtained with 3 projections and only a very small loss of resolution appears in the layer structure, when compared with the 21-projection reconstruction (not shown here). This is obviously not the case for the highly elliptical preform of Figs. 4(d) and 5(d). Here the reconstruction with 7 projections gives rise to a maximum error of about 30 percent and the profile shape obtained with 3 projections is no longer recognizable.

\section{Applicability of Marcuse's Equation}

We refer the reader to Section III-D where the characteristics of Marcuse's equation have been stressed and we present here the results obtained. For the reason given in Section III-D, we consider only the errors made in the index difference and in the index at the center of the core; the supposed exact values of these two parameters were obtained from Chu's reconstruction with 21 projections (as before, the errors are normalized by the index difference of the preform). We consider again the cases of preforms $B$ and $C$; Table VI shows the maximum error made in the results together with the improvement provided by the averaging technique described in Section III-D. In column I only one projection is measured and the average is performed on the results obtained from the two halves, $\rho \leqslant$ 0 and $\rho \geqslant 0$, of the deflection. In columns II and III, 3 and 7 projections are measured and the average is thus made on 6 and 14 quantities, respectively.

From Table VI, we see that the preforms $B$ and $C$ do not satisfy our arbitrary accuracy requirement of 5 percent but column I shows that, in the case of averaging, preform $B$ does. When the average involves three or seven projections (columns II and III), the error decreases accordingly. A comparison with the results of Table $V$ stresses the fact that, for three projections the resulting error is three (preform $C$ ) to ten (preform $B$ ) times larger than if these three projections had been used in a reconstruction with Chu's equation.

For the routine measurement of "every-day" near-circular preforms, the systematic use of Chu's equation with three projections thus seems very tempting if one is only interested in the accuracy achieved. However, the limit of applicability of (9) can be extended if a higher error level is tolerated and if one is mainly concerned with the speed of the calculations [5 min with (9)]. As it may be a determining factor in the choice of the algorithm, we turn now to the reconstruction time by (6) and (9). As mentioned above, the calculation of 
TABLE VI

Accuracy of Marcuse's Equation and Effects of Averaging Column I: 1 Measured Projection Average of $\Delta_{L}$ and $\Delta_{R}$ Column II: 3 Measured Projections, Average of 6 Profiles Column III: 7 Measured Projections, Average of 14 Profiles

\begin{tabular}{|c|c|c|c|c|c|}
\cline { 2 - 6 } \multicolumn{1}{c|}{} & & $\begin{array}{c}\text { Maximm error } \\
\text { in }\end{array}$ & \multicolumn{4}{|c|}{ Maximm error in $\Delta$ when averaging } \\
\hline \multirow{4}{*}{$\mathrm{n}(0,0)$} & $\mathrm{B}$ & 8.4 & 1.5 & 1.2 & 0.4 \\
\cline { 2 - 6 } & $\mathrm{C}$ & 12 & 5.2 & 4.5 & 1.2 \\
\hline \hline \multirow{3}{*}{$\Delta$} & $\mathrm{B}$ & 10 & 3.8 & 2.8 & 0.4 \\
\cline { 2 - 6 } & $\mathrm{C}$ & 14.8 & 5.6 & 4.9 & 1 \\
\hline
\end{tabular}

the convolution $g(z, \theta)$ in (6) is performed very quickly and all the computing time is spent in the integration. The integration needs interpolated values of $g(z, \theta)$ both in the $z$ and in the $\theta$-direction, whereas (9) uses the deflection values without any interpolation. As the number of integration points are nearly identical in both equations, the ratio of the computing times by (9) and (6) is believed to be the ratio of the access times for an interpolated value of $g(z, \theta)$ and a value of $\phi(\rho)$. In our case, for the interpolation routine described at the end of Section II, this ratio was evaluated to be about 30 . The implementation of Chu's reconstruction would take therefore about $2 \mathrm{~h} 30 \mathrm{~min}$ on Tektronix 4052 (instead of $5 \mathrm{~min}$ ). Oh. viously, it is possible to reduce that time by using an interpolation involving less than 18 points. To conclude this section, we would like to stress that during the calculation the computer does not need any external intervention and thus a long computing time is perhaps not too serious a problem in practice.

\section{Conclusions}

This paper has considered the practical implementation of the 3-D reconstruction of preform index profiles from the knowledge of the deflection function. The spatial filtering technique of measuring the deflection function has been used when the case of real preforms was considered. We have investigated the influence on the reconstruction quality of the scan increment $\epsilon$, the number $N_{I}$ of points in the numerical integration involved, and the number $N_{\theta}$ of projections used. Special emphasis has been placed on $N_{\theta}$ as this parameter determines the algorithm used in the reconstruction. The importance of using a sufficient number of points for the integration involved in preform profile reconstruction has been demonstrated with both simulated and measured profiles. The systematic use of interpolation enables the optimum number of integration points to be obtained from the data corresponding to a few preform projections. The storage requirements corresponding to three projections are within the memory capacity of a laboratory minicomputer, thus avoiding the problem of data transfer to a large computer. The procedure therefore provides an efficient tool in the routine evaluation of nearcircular preforms. For highly asymmetric preforms however, as we have shown in an example, no simplified solution is possible and a complete tomographic reconstruction must be used.

\section{APPENDIX A}

\section{Relation Between Chu's and Marcuse's Equations}

In [1] Chu expresses the reconstruction equation in the form of (2) and a convergence problem arises when $\rho=r \sin (\psi-\theta)$, which always happens in the $\rho$-integration (for $r \leqslant$ preform radius). On the other hand, Chu recognizes a Hilbert transform in the $\rho$-integration [1]:

$$
g(z, \theta)=\int_{-\infty}^{+\infty} \frac{\partial \eta(\rho, \theta)}{\partial \rho} \frac{d \rho}{z-\rho} .
$$

All convergence problems disappear when one considers the rigorous formula for the Hilbert transform which gives

$$
g(z, \theta)=V p\left\{\int_{-\infty}^{+\infty} \frac{\partial \eta(\rho, \theta)}{\partial \rho} \frac{d \rho}{z-\rho}\right\} .
$$

The notation $V p\{\}$ signifies that the Cauchy principal part of the integral in the bracket is to be taken.

We can now derive Marcuse's equation (9) from Chu's equation (5) for the case of a perfectly circular preform in which the explicit $\theta$-dependence of $\phi(\rho, \theta)$ disappears.

In this case, $(5)$ reduces to

$$
\frac{n(r)-n_{o}}{n_{o}}=-\frac{1}{2 \pi^{2}} \int_{-\pi / 2}^{+\pi / 2} d \theta V p\left\{\int_{-\infty}^{\infty} \phi(\rho) \frac{d \rho}{r \sin \theta+\rho}\right\}
$$

or, by interchanging the $\rho$ and $\theta$ integrations,

$$
\frac{n(r)-n_{o}}{n_{o}}=-\frac{1}{2 \pi^{2}} \int_{-\infty}^{+\infty} \phi(\rho) d \rho V p\left\{\int_{-\pi / 2}^{+\pi / 2} \frac{d \theta}{r \sin \theta+\rho}\right\}
$$

We must evaluate the Cauchy principal part of the integral $I$ :

$$
I=\int_{-\pi / 2}^{+\pi / 2} \frac{d \theta}{r \sin \theta+\rho}=\int_{-1}^{+1} \frac{2 d t}{\rho t^{2}+2 r t+\rho}=I_{[-1,+1]}
$$

with $t=\tan \theta / 2$.

From the relative values of $|\rho|$ and $r$, we distinguish three cases.

1) $r<|\rho|$ : The function has no poles and

$$
\begin{array}{r}
I=\operatorname{sgn}(\rho) \frac{\pi}{\sqrt{\rho^{2}-r^{2}}} \quad \text { where } \operatorname{sgn}(\rho)=+1 \text { if } \rho \geqslant 0 \\
\cdot \operatorname{sgn}(\rho)=-1 \text { if } \rho<0 .
\end{array}
$$

2) $r=\rho$ : There is a double pole at $t=-1$, and $I=\infty$. This case can be contained in the analytical continuation of case 1 ).

3) $r>|\rho|$ : There are two poles $t_{1} \neq t_{2}$ and to ensure convergence we must divide the integration interval into three and consider the integral family $\left\{I_{\alpha}\right\}_{\alpha \text { small }}$

$$
I_{\alpha}=I_{\left[-1, t_{1}-\alpha\right]}+I_{\left[t_{1}+\alpha, t_{2}-\alpha\right]}+I_{\left[t_{2}+\alpha, 1\right]} \text {. }
$$

The principal part of $I$ corresponds to the limit of the $\left\{I_{\alpha}\right\}_{\alpha}$ when $\alpha$ decreases toward 0 and the result is $\lim I_{\alpha}=0$. 
As a conclusion, (A4) reduces to

$$
\begin{aligned}
\frac{n(r)-n_{o}}{n_{o}} & =-\frac{1}{2 \pi} \int_{r<|\rho|} \phi(\rho) \operatorname{sgn}(\rho) \frac{d \rho}{\sqrt{\rho^{2}-r^{2}}} \\
& =-\frac{1}{\pi} \int_{r}^{\infty} \frac{1}{2}(\phi(\rho)-\phi(-\rho)) \frac{d \rho}{\sqrt{\rho^{2}-r^{2}}} .
\end{aligned}
$$

In the case of a perfectly circular preform, we have $\phi(\rho)=$ $-\phi(-\rho)$ and (5) reduces to Marcuse's equation (9). The same derivation shows that (9) reduces to (8). In the case of a quasicircular preform, the deflection is no longer antisymmetric but (A6) justifies, to some extent, making an average of the profiles obtained when (9) is applied to the left and the right sides of the deflection.

\section{APPENDIX B}

\section{Elliptical Preform with a Parabolic Profile}

With the assumption of small numerical aperture, the pathlength difference $\eta(\rho, \theta)$ and the deflection $\phi(\rho, \theta)$ are related by (4):

$$
\frac{\partial \eta(\rho, \theta)}{\partial \rho} \simeq n_{o} \phi(\rho, \theta) \text {. }
$$

We apply this approximate relation to the case of an elliptical preform with parabolic index

$$
\begin{array}{rlrl}
n(x, y) & =n_{o}\left\{1+\Delta\left(1-\left[\left(\frac{x}{a}\right)^{2}+\left(\frac{y}{b}\right)^{2}\right]\right)\right\}, & \\
& =n_{0}, & \text { for }\left(\frac{x}{a}\right)^{2}+\left(\frac{y}{b}\right)^{2} \leqslant 1 \\
& \text { for }\left(\frac{x}{a}\right)^{2}+\left(\frac{y}{b}\right)^{2}>1
\end{array}
$$

and obtain

$$
\begin{aligned}
& \phi(\rho, \theta) \simeq \frac{\partial}{\partial \rho} \int_{A B^{\prime}} \frac{n(x, y)-n_{o}}{n_{o}} d s \\
& \simeq \Delta \frac{a b}{R_{\theta}^{2}}\left(-\frac{4 \rho}{R_{\theta}}\right) \sqrt{1-\left(\frac{\rho}{R_{\theta}}\right)^{2}}, \\
& \left.\begin{array}{ll}
=0 & \text { for }|\rho| \leqslant R_{\theta} \\
\text { for } & |\rho|>R_{\theta}
\end{array}\right\}
\end{aligned}
$$

where $R_{\theta}=\sqrt{a^{2} \sin ^{2} \theta+b^{2} \cos ^{2} \theta}$ is the apparent radius when the preform is seen in the direction $\theta$ [notation as in Fig. 2(a)], and $A B^{\prime}$ is the straight path of Fig. 1 (as an approximation for the actual one $A B$ ).

The $\theta$-dependence occurs only via the intermediary of the apparent radius $R_{\theta} . R_{\theta}$ enters as scaling factor for the distance $\rho$ and in the dimensionless factor $a b / R_{\theta}^{2}$, which multiplies the index difference $\Delta$. These observations coincide with the conclusions of [7] and [8]. The circular preform "equivalent" to an elliptically deformed one has a radius equal to $R_{\theta}$ and an index difference equal to $\Delta a b / R_{\theta}^{2}$.
Marcuse's equation (9) applied, for a particular $\theta$, to the deflection $\phi(\rho, \theta)$ expressed by (B2) yields the following circular profile:

$$
\frac{n(r)-n_{o}}{n_{o}}=\Delta \frac{a b}{R_{\theta}^{2}}\left(1-\frac{r^{2}}{R_{\theta}^{2}}\right) .
$$

The error in index-difference is then

$$
\text { error }(\Delta)=\frac{a b}{R_{\theta}^{2}}-1
$$

which, with the notation of Section III can be expressed as

$$
\text { error }(\Delta)=e \cos 2 \theta^{\prime}+e^{2} \cos \theta^{\prime} \cos 3 \theta^{\prime}+0\left(e^{3}\right)
$$

where $e=(b-a) / b$ and $\theta^{\prime}$ is the scan-direction.

The error distribution of (B5) is shown in Fig. 3(a).

\section{ACKNOWLEDGMENT}

The authors wish to thank E. J. Tarbox, R. J. Mansfield, and R. Birch for providing the preforms used in this study, D. N. Payne for instigating and encouraging the work, and Prof. W. A. Gambling for his guidance. The French Post Office is gratefully acknowledged for the award of a research fellowship.

\section{REFERENCES}

[1] P. L. Chu and T. Whitbread, "Nondestructive determination of refractive index profile of an optical fiber: Fast Fourier transform method," Appl. Opt., vol. 18, pp. 1117-1122, Apr. 1979.

[2] I. Sasaki, D. N. Payne, and M. J. Adams, "Measurement of refractiveindex profiles in optical-fibre preforms by spatial-filtering technique," Electron. Lett., vol. 16, pp. 219-221, Mar. 13, 1980.

[3] I. Sasaki, P. L. Francois, and D. N. Payne, "Accuracy and resolution of preform index-profiling by the spatial-filtering method," presented at the 7th ECOC, Copenhagen, Denmark, Sept. 1981.

[4] C. Saekeang, "Determination of optical fibre parameters," Ph.D. dissertation, Univ. New South Wales, Sydney, Australia, Mar. 1980.

[5] P. L. Chu and C. Saekeang, "Nondestructive determination of refractive-index profile and cross-sectional geometry of opticalfibre preform," Electron. Lett., vol. 15, pp. 635-637, Sept. 27, 1979.

[6] P. L. Chu, "Nondestructive refractive-index profile measurement of elliptical optical fibre or preform," Electron. Lett., vol. 15, pp. 357-358, June 7, 1979.

[7] D. Marcuse, Principles of Optical Fibre Measurements. New York: Academic, 1981.

[8] K. F. Barrell and C. Pask, "Nondestructive index profile measurement of noncircular optical fibre preforms," Opt. Commun., vol. 27, pp. 230-234, Nov. 1978.

[9] M. A bramowitz and I. A. Stegun, Handbook of Mathematical Functions. New York: Dover, 1965

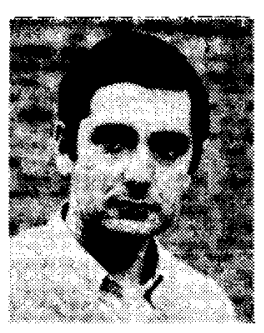

Pierre-Luc Francois was born in France in 1955. He graduated from Ecole Polytechnique of Paris in 1978 and the Ecole Nationale Superieure des Telecommunications in 1980.

He then joined the Centre National d'Etudes des Telecommunications (CNET), Lannion, France where he works in the department concerned with propagation characteristics of monomode fibers. He has just spent one year at the University of Southampton, Hampshire, England. 


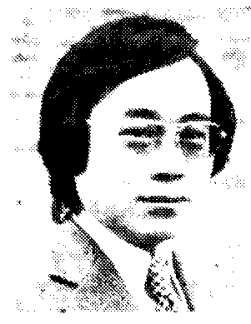

Issei Sasaki was born in Japan in 1947. He graduated from Mororan Institute of Technology, Hokkaido, Japan, in 1970

From 1970 to 1978 he was a Research Associate at Hokkaido University and since 1978 he has been engaged in research on optical fibers at the University of Southampton, Hampshire, England.

Mr. Sasaki is a member of the Institute of Electronics and Communication Engineers of Japan.

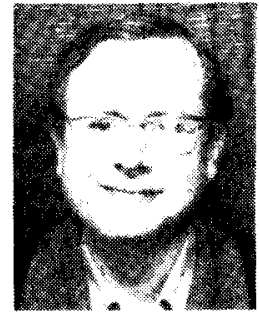

M. J. Adams graduated from Imperial College, London University, London, England, in 1966 and received the M.Sc. and Ph.D. degrees from the University of Wales in 1967 and 1970, respectively.

After three years as a Research Fellow at University College, Cardiff, Wales, he worked from 1973 to 1975 at Plessey Radar, Cowes, Isle of Wight. Since 1975 he has been employed at the University of Southampton, Hampshire, Fngland, where he is now a Lecturer in the Department of Electronics. His research interests include theoretical aspects of optical fibers and semiconductor optical components. He is the author or coauthor of over 50 papers and has recently published a book on optical waveguides. 